\title{
Improving the Dynamic Performance of Distribution Electronic Power Transformers Using Sliding Mode Control
}

\author{
Rahmat-Allah Hooshmand ${ }^{\dagger}$, Mohammad Ataei*, and Mohammad Hosein Rezaei** \\ $\dagger^{*}$ Dept. of Electrical Eng., University of Isfahan, Isfahan, Iran \\ ** Dept. of Electrical Eng., Islamic Azad University, Najaf Abad Branch, Najaf Abad, Iran
}

\begin{abstract}
These days, the application of electronic power transformers (EPTs) is expanding in place of ordinary power transformers. These transformers can transmit power via three or four wire converters. Their dynamic performance is extremely important, due to their complex structure. In this paper, a new method is proposed for improving the dynamic performance of distribution electronic power transformers (DEPT) by using sliding mode control (SMC). Hence, to express the dynamic characteristics of a system, different factors such as the voltage unbalance, voltage sag, voltage harmonics and voltage flicker in the system primary side are considered. The four controlling aims of the improvement in dynamic performance include: 1) maintaining the input currents so that they are in sinusoidal form and in phase with the input voltages so they have a unity power factor, 2) keeping the dc-link voltage within the reference amount, 3) keeping the output voltages at a fixed amount and 4) keeping the output voltages in sinusoidal and symmetrical forms. Simulation results indicate the potential and capability of the proposed method in improving DEPT behavior.
\end{abstract}

Key Words: Distribution electronic power transformer (DEPT), Dynamic performance, Linear quadratic regulator (LQR), PI controller, Sliding mode control (SMC) and State space model

\section{INTRODUCTION}

Some of the essential parts of an electric distribution system are the transformers which are responsible for duties like voltage transmission and isolation. Nevertheless, they are one of the heaviest and most expensive parts of low frequency $(50 \mathrm{~Hz})$ distribution systems. Nowadays, EPTs are used instead of ordinary power transformers to fulfill voltage transmission and to deliver power to the power system by using power electronic converters [1], [2].

The main advantage of an EPT is its ability to control the voltage phase angle and amplitude in both the primary and secondary sides, immediately, via power electronic converters [3].

It is known that saturation flux density has a reverse relation with frequency. Therefore, by using a high frequency link via an EPT, the bulky iron core and heavy copper windings in transformers can be eliminated.

In addition to the mentioned duties, this type of transformer improves various qualities of the power, including the voltage unbalances, voltage harmonics, voltage sags, voltage swells

\footnotetext{
Manuscript received Apr. 20, 2011; revised Nov. 19, 2011

Recommended for publication by Associate Editor Jun-Keun Ji.

$\dagger$ Corresponding Author: Hooshmand_r@eng.ui.ac.ir

Tel: +98-311-7934073, Fax: +98-311-7933071, University of Isfahan

* Dept. of Electrical Eng., University of Isfahan, Iran

** Dept. of Electrical Eng., Najaf Abad Branch, Islamic Azad University, Iran
}

and voltage fluctuations in the distribution network, as well as the voltage control, power flow control, static and dynamic stability improvements in the transmission network [4]-[8].

EPTs have received a great deal of attention in recent years in both industry and scientific centers and many studies have been performed on their performance mechanism, structural design, parallel performance and control strategies [9]-[12].

In terms of the performance mechanism, first an EPT is analyzed as a single-phase structure with connected converters in series, and then the switching of the converter in foursteps is dealt with [9]. The EPT structure is also considered in terms of structural design, and then an appropriate design for the considered system is proposed, in order to achieve the system requirements [10], [11]. Also, the subject of parallel performance is considered. The EPT performance is compared with that of an ordinary power transformer and an appropriate control strategy is used [12].

But the main application studies focus on EPT performance in distribution systems (DEPT). There are two main structures for DEPT; one does not have a DC-link [8], [13] and the other has a DC-link [7], [12]. Using a DC-link has advantages such as controllable voltage and current on both sides of the DEPT via pulse width modulation (PWM) technology. By utilizing SMC, a new method is presented in this paper for improving the DEPT dynamic behavior with a DC-link. This dynamic 
improvement is considered in terms of the following four controlling aims:

1) Maintaining the input currents in a sinusoidal form and in phase with the input voltages having a unity power factor.

2) Keeping the dc-link voltage within the reference amount.

3) Maintaining the output voltages at fixed amounts.

4) Keeping the output voltages in sinusoidal and symmetrical forms.

The DC-link voltage in the input stage plays an important role in achieving the four controlling aims. Therefore, in section 2, a DEPT model with a 3-wire DC-link is considered. In the third section, the control and proper performance of the DEPT are analyzed by designing a PI controller and a linear quadratic regulator (LQR), and then introducing sliding mode control (SMC). In section 4, the method of using the proposed SMC in the DEPT is presented. The simulation results in the fifth section show the capability of the SMC method, in comparison with the PI and LQR methods.

\section{DEPT MODEL}

In this section, the DEPT model is expressed in terms of the DC-link existence. Fig. 1 shows a diagram for a DEPT base with a DC-link, which includes two power electronic converters in the primary and secondary side and a medium frequency transformer (MFT).

The main task of a MFT is the isolation of the system primary and secondary side. Since the size of a transformer has inverse relation with the frequency, a medium frequency transformer (with a frequency of $1 \mathrm{kHz}$ ) is smaller than an ordinary power transformer (with a frequency of $50 \mathrm{~Hz}$ ). According to Fig. 1, first a sinusoidal input voltage with a frequency of $50 \mathrm{~Hz}$ is converted into a medium frequency signal $(1 \mathrm{kHz})$ and then it is coupled magnetically towards the secondary side. In the secondary side, the medium frequency signal is transformed into a sinusoidal output voltage via a converter.

Fig. 2 shows a DEPT structure with a 3-wire DC-link. As can be seen, this transformer is formed by three-stages that include the input, isolation and output stages. The input stage is a three-phase PWM rectifier that converts a threephase sinusoidal input voltage into DC voltage. The isolation stage includes an H-bridge-1 converter, a medium frequency transformer (MFT) and an H-bridge-2 converter. The obtained DC voltage from the input stage feeds the H-bridge- 1 and is modulated into a medium frequency square waveform. Then the obtained square wave is delivered to the MFT and the rectifier and converted into a DC voltage via the H-bridge-2. There is a three-phase high frequency inverter in the output stage that transforms the obtained DC voltage of the isolation stage into a three-phase ac sinusoidal voltage.

Fig. 3 shows a simplified diagram of a DEPT. The transient states of the H-bridge are so fast in this simplified diagram that they can be neglected. Also, the leakage inductance of the MFT is sufficiently small in the real condition that it can be ignored.
According to Fig. 2, the dynamic equations include:

$$
\begin{gathered}
\left\{\begin{array}{r}
L \frac{d i_{l a}(t)}{d t}=u_{s a}(t)-u_{l a}(t)+R i_{l a}(t) \\
L \frac{d i_{l b}(t)}{d t}=u_{s b}(t)-u_{l b}(t)+R i_{l b}(t) \\
L \frac{d i_{l c}(t)}{d t}=u_{s c}(t)-u_{l c}(t)+R i_{l c}(t)
\end{array}\right. \\
\left\{\begin{array}{c}
C_{f} \frac{d u_{L a}(t)}{d t}=i_{f a}(t)-i_{L a}(t) \\
C_{f} \frac{d u_{L b}(t)}{d t}=i_{f b}(t)-i_{L b}(t) \\
C_{f} \frac{d u_{L c}(t)}{d t}=i_{f c}(t)-i_{L c}(t) \\
L_{f} \frac{d i_{f a}(t)}{d t}=\frac{u_{o a}(t)}{k}-u_{L a}(t) \\
L_{f} \frac{d i_{f b}(t)}{d t}=\frac{u_{o b}(t)}{k}-u_{L b}(t) \\
L_{f} \frac{d i_{f c}(t)}{d t}=\frac{u_{o c}(t)}{k}-u_{L c}(t)
\end{array}\right.
\end{gathered}
$$

$$
\begin{gathered}
\frac{d}{d t}\left(\frac{1}{2} C u_{d c}^{2}\right)=u_{l a}(t) i_{l a}(t)+u_{l b}(t) i_{l b}(t)+u_{l c}(t) i_{l c}(t) \\
-\frac{u_{o a}(t) i_{f a}(t)}{k}-\frac{u_{o b}(t) i_{f b}(t)}{k}-\frac{u_{o c}(t) i_{f c}(t)}{k}
\end{gathered}
$$

where,

$$
C=C_{1}+C_{2} / k^{2}
$$

$L, R$ : inductor and resistor of the input stage

$C_{1}, C_{2}:$ dc capacitors of the input and output stages

$L_{f}, C_{f}$ : filter inductor and capacitor of the output stage

$k:$ transformation ratio of MFT

$U_{d c}$ : DC-link voltage of the input stage

c.b.a: denotes three-phase stationary reference frame

$u_{s}=\left[u_{s a}, u_{s b}, u_{s c}\right]:$ instantaneous input voltages

$u_{L}=\left[u_{L a}, u_{L b}, u_{L c}\right]:$ instantaneous output voltages

$u_{l}=\left[u_{l a}, u_{l b}, u_{l c}\right]:$ instantaneous ac terminal voltages of the input stage

$u_{o}=\left[u_{o a}, u_{o b}, u_{o c}\right]:$ instantaneous ac terminal voltages of the output stage

$$
i_{l}=\left[i_{l a}, i_{l b}, i_{l c}\right]: \text { input currents }
$$

$i_{f}=\left[i_{f a}, i_{f b}, i_{f c}\right]:$ filter inductance currents of the output stage

$i_{L}=\left[i_{L a}, i_{L b}, i_{L c}\right]:$ load currents

In addition to these equations, the voltage variables regarding equations (1) to (4) can be expressed with the following algebraic equations:

$$
\begin{gathered}
\left\{\begin{array}{c}
u_{s a}(t)=\sqrt{2} U_{s} \sin \omega t \\
u_{s b}(t)=\sqrt{2} U_{s} \sin \left(\omega t-\frac{2 \pi}{3}\right) \\
u_{s c}(t)=\sqrt{2} U_{s} \sin \left(\omega t+\frac{2 \pi}{3}\right)
\end{array}\right. \\
\left\{\begin{array}{l}
u_{l a}(t)=m_{1} U_{d c} \sin \left(\omega t-\theta_{1}\right) \\
u_{l b}(t)=m_{1} U_{d c} \sin \left(\omega t-\theta_{1}-\frac{2 \pi}{3}\right) \\
u_{l c}(t)=m_{1} U_{d c} \sin \left(\omega t-\theta_{1}+\frac{2 \pi}{3}\right)
\end{array}\right.
\end{gathered}
$$




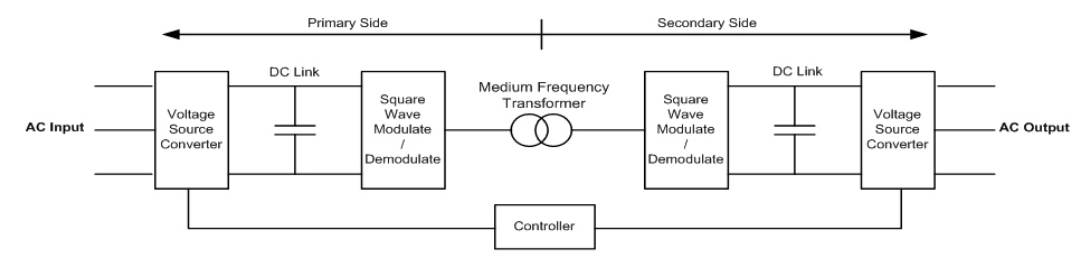

Fig. 1. The diagram for DEPT base with DC-link.

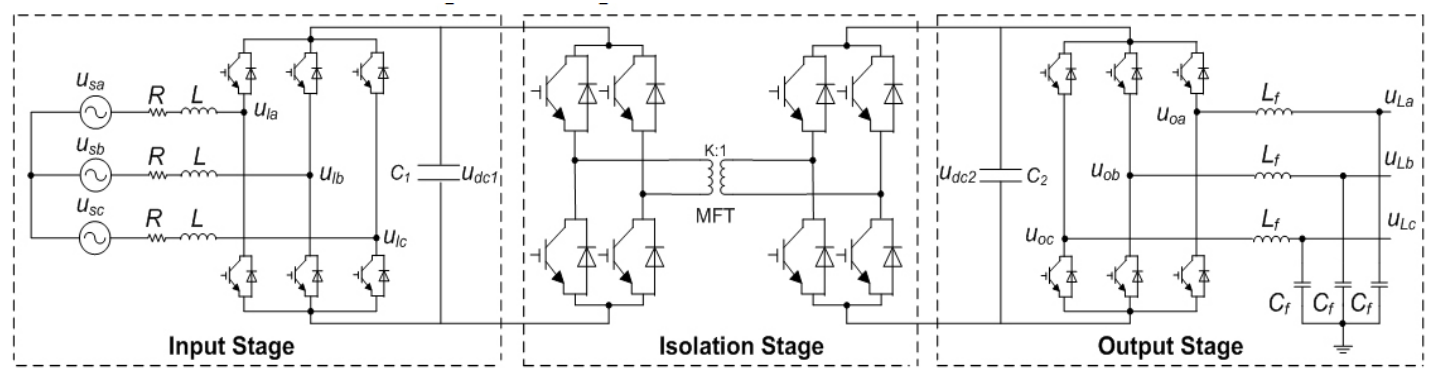

Fig. 2. The DEPT structure with 3-wire DC-link.

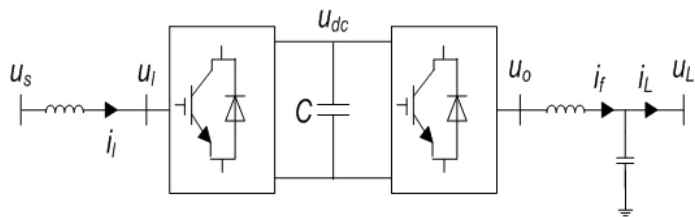

Fig. 3. Simplified diagram of 3-wire DEPT.

$$
\left\{\begin{array}{l}
u_{o a}(t)=m_{2} U_{d c} \sin \left(\omega t-\theta_{2}\right) \\
u_{o b}(t)=m 2 U_{d c} \sin \left(\omega t-\theta_{2}-\frac{2 \pi}{3}\right) \\
u_{o c}(t)=m_{2} U_{d c} \sin \left(\omega t-\theta_{2}+\frac{2 \pi}{3}\right)
\end{array}\right.
$$

where,

$\omega$ : modulation frequency

$m_{1}, \theta_{1}$ : modulation index and angle of the sinusoidal modulating waveform of the converter in the input stage

$m_{2}, \theta_{2}$ : modulation index and angle of the sinusoidal modulating waveform of the converter in the output stage

In equations (1) to (4), the DEPT dynamic differential equations factors are varied with time and should be taken to the synchronously rotating reference frame via Park's transformer, in order to get invariable equations according to time [14]. Therefore, the four dynamic equations of (1) to (4) in the d-q rotating reference frame are as follows:

$$
\begin{gathered}
\left\{\begin{array}{c}
\frac{d i_{l d}}{d t}=-\frac{R}{L} i_{l d}-\omega i_{l q}+\frac{m_{1} \sin \theta_{1}}{L} u_{d c} \\
\frac{d i_{l q}}{d t}=-\frac{R}{L} i_{l q}+\omega i_{l d}-\frac{m_{1} \cos \theta_{1}}{L} u_{d c}+\sqrt{2} \frac{u_{s}}{L}
\end{array}\right. \\
\left\{\begin{array}{c}
\frac{d u_{L d}}{d t}=\frac{1}{C_{f}} i_{f d}-\frac{1}{C_{f}} i_{L d}-\omega u_{L q} \\
\frac{d u_{L q}}{d t}=\frac{1}{C_{f}} i_{f q}-\frac{1}{C_{f}} i_{L q}+\omega u_{L d}
\end{array}\right. \\
\left\{\begin{array}{l}
\frac{d i_{f d}}{d t}=-\omega i_{f d}-\frac{m_{2} \sin \theta_{2}}{k L_{f}} u_{d c}-\frac{1}{L_{f}} u_{L d} \\
\frac{d i_{f q}}{d t}=\omega i_{f d}+\frac{m_{2} \cos \theta_{2}}{k L_{f}} u_{d c}-\frac{1}{L_{f}} u_{L q}
\end{array}\right.
\end{gathered}
$$

$$
\begin{gathered}
\frac{d u_{d c}}{d t}=-\frac{3 m_{1}}{2 C} i_{l d} \sin \theta_{1}+\frac{3 m_{1}}{2 C} i_{l q} \cos \theta_{1} \\
+\frac{3 m_{2}}{2 k C} i_{f d} \sin \theta_{2}-\frac{3 m_{2}}{2 k C} i_{f q} \cos \theta_{2}
\end{gathered}
$$

where,

$q, d$ : denotes rotating reference frame

$\left[i_{l d}, i_{l q}\right]:$ input currents

$\left[i_{f d}, i_{f q}\right]:$ filter inductance currents

$\left[i_{L d}, i_{L q}\right]:$ load currents

$\left[u_{L d}, u_{L q}\right]$ : output voltages

It should be noted that according to Park's transformer, the following is obtained:

$$
\begin{aligned}
& {\left[i_{l d} i_{l q} i_{l 0}\right]^{T}=K\left[i_{l a} i_{l b} i_{l c}\right]^{T},\left[i_{L d} i_{L q} i_{L 0}\right]^{T}=K\left[i_{L a} i_{L b} i_{L c}\right]^{T}} \\
& ,\left[i_{f d} i_{f q} i_{f 0}\right]^{T}=K\left[i_{f a} i_{f b} i_{f c}\right]^{T},\left[u_{L d} u_{L q} u_{L 0}\right]^{T}=K\left[u_{L a} u_{L b} u_{L c}\right]^{T}
\end{aligned}
$$

$$
K=\frac{2}{3}\left[\begin{array}{ccc}
\cos \omega t & \cos \left(\omega t-\frac{2 \pi}{3}\right) & \cos \left(\omega t+\frac{2 \pi}{3}\right) \\
\sin \omega t & \sin \left(\omega t-\frac{2 \pi}{3}\right) & \sin \left(\omega t+\frac{2 \pi}{3}\right) \\
\frac{3}{2} & \frac{3}{2} & \frac{3}{2}
\end{array}\right]
$$

\section{DEPT CONTROLlERs StruCture}

To achieve the aims mentioned in the introduction, the input, isolation and the output stages should be controlled in a suitable way. This section deals with designing the LQR and the PI controllers and introducing the SMC. Also, separate controllers are considered for each of the three-stage of the PI controllers. However, the LQR and SMC, controllers are only designed for input and output stages.

\section{A. PI controller for a DEPT}

For controlling a DEPT and for achieving a more appropriate performance, the following controlling method, based on a PI controller for each of the input, isolation and output stages, is used [10]. 


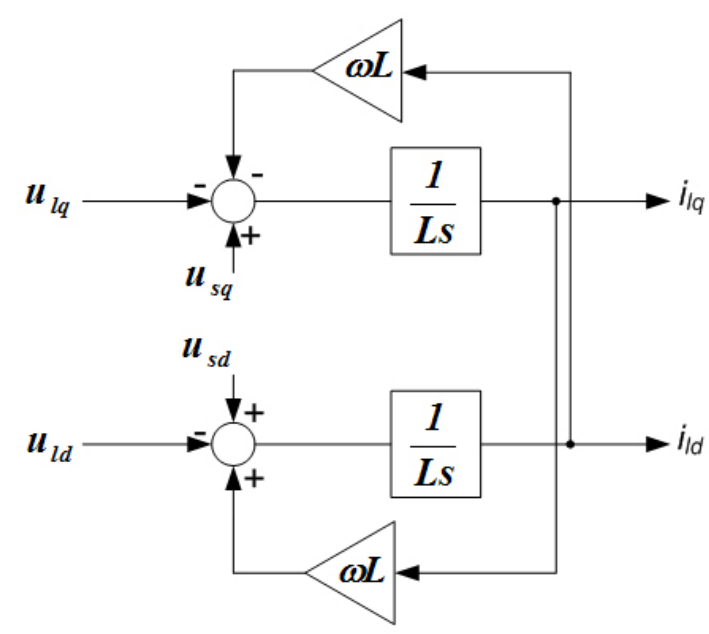

Fig. 4. Model of the input stage.

1) Control method for the input stage: The input stage is connected directly to the primary of the system. In order to prevent harmonic injection into the network, the input current should be sinusoidal and in phase with the input voltage and the power factor should equal to "1". Thus, a controllable current source for controlling the input stage is used.

The mathematical model of the input stage can be written in the a-b-c stationary reference frame or in the d-q rotating reference frame. However, using the controlling method in the d-q frame results in better performance [14]. Hence, the mathematical model of the input stage in the $d-q$ rotating reference frame can be used as follows:

$$
\left\{\begin{array}{l}
L \frac{d i_{l d}}{d t}=\omega L i_{l q}-u_{l d}+u_{s d} \\
L \frac{d i_{l q}}{d t}=-\omega L i_{l d}-u_{l q}+u_{s q}
\end{array}\right.
$$

where the existing variables in the equations include:

$$
\begin{gathered}
{\left[\begin{array}{ll}
i_{l d} & i_{l q}
\end{array}\right]^{T}=K\left[\begin{array}{lll}
i_{l a} & i_{l b} & i_{l c}
\end{array}\right]^{T}} \\
{\left[\begin{array}{ll}
u_{l d} & u_{l q}
\end{array}\right]^{T}=K\left[\begin{array}{lll}
u_{l a} & u_{l b} & u_{l c}
\end{array}\right]^{T}} \\
{\left[\begin{array}{ll}
u_{s d} & u_{s q}
\end{array}\right]^{T}=K\left[\begin{array}{lll}
u_{s a} & u_{s b} & u_{s c}
\end{array}\right]^{T}} \\
K=\frac{2}{3}\left[\begin{array}{lll}
\sin \omega t & \sin (\omega t-120) & \sin (\omega t+120) \\
\cos \omega t & \cos (\omega t-120) & \cos (\omega t+120)
\end{array}\right]
\end{gathered}
$$

$\omega:$ synchronous angular velocity of the grid voltage

$\left[i_{l d}, i_{l q}\right]$ : input currents in the synchronous rotating frame

$\left[u_{l d}, u_{l q}\right]:$ terminal voltages of input stage in the synchronous rotating frame

$\left[u_{s d}, u_{s q}\right]:$ input voltages in the synchronous rotating frame

$K$ : Park's transformation matrix

$i_{l}=\left[i_{l a}, i_{l b}, i_{l c}\right]:$ input currents in the stationary frame

$u_{l}=\left[u_{l a}, u_{l b}, u_{l c}\right]:$ terminal voltages of input stage in the stationary frame

$u_{s}=\left[u_{s a}, u_{s b}, u_{s c}\right]:$ input voltages in the stationary frame

This model is shown in Fig. 4.

Equation (12) and Fig. 4 show that there is a relation between the "d" and "q" axes that could affect the dynamic performance of the system. To solve this problem and separate the d-q voltage, the components of the control input source voltage are used as a "feed-forward". To keep the dc voltage fixed and the input current sinusoidal, a double loop control is used, where one loop is a dc voltage output loop and the other is a current internal loop. This method is shown in Fig. 5.

The starred variables in this figure indicate the reference variables. As can be seen in Fig. 5, $i_{l d}^{*}$ is obtained from the dc voltage outer loop and $i_{l q}^{*}$ is found from the power factor loop. Thus, for a power factor to equal " 1 ", $i_{l q}^{*}$ tends to zero. If the $\mathrm{d}$ axis with the network voltage is considered to be in one direction, $e_{q}=0$ is reached. Therefore, according to Fig. 5 , equation (12) could be simplified as follows:

$$
\left\{\begin{array}{l}
L \frac{d i_{l d}}{d t}=\left(K_{p}+\frac{K_{I}}{s}\right)\left(i_{l d}^{*}-i_{l d}\right) \\
L \frac{d i_{l q}}{d t}=\left(K_{p}+\frac{K_{I}}{s}\right) i_{l q}
\end{array}\right.
$$

where, $K_{p}, K_{I}$ are control coefficients.

2) Control method for the isolation stage: In the isolation stage, the DC voltage obtained from the input stage is modulated into a medium frequency square wave and is coupled to the secondary side. Then, the resultant voltage is converted into a DC voltage.

Here, the task of the medium frequency transformer is isolation, as well as the control of voltage level fluctuations. An electronic power converter can change the voltage level directly, but there is enormous pressure on semiconductor equipment due to high frequencies [15].

To simplify the design of the control system, an open loop PWM controller is applied for the H-bridge- 1 converter. If the power transmission is wanted only in one direction, then a diode rectifier should be used. Hence, a proportional amplifier can be used for the isolation stage. A simplified model of this is shown as follows:

$$
V_{d c 2}=\frac{1}{k} V_{d c 1}
$$

where, $k$ is transformation ratio of the MFT, and $V_{d c 1}$ and $V_{d c 2}$ are the dc bus voltages of the input and output stages, respectively.

3) Control method for the output stage: The output is directly connected to the consumer. Therefore, its output voltage should be a fixed amount. As mentioned earlier, there are three single-phase inverters in this stage and each inverter has its own separate output phase. Hence, controlling each one can be done independently. In order to connect a load in the output, the inverter should be controlled as a single-phase sinusoidal voltage source.

As can be seen, when the switching frequency is larger than the reference signal, the inverter can be controlled as a proportional amplifier. Therefore, the mathematical model for the output stage is defined as follows:

$$
\left\{\begin{array}{c}
C_{f} \frac{d u_{L}}{d t}=i_{f}-i_{L} \\
L_{f} \frac{d i_{f}}{d t}=u_{o}-u_{L}
\end{array}\right.
$$

where the parameters of the equation are as defined previously after equations (1) to (4). 


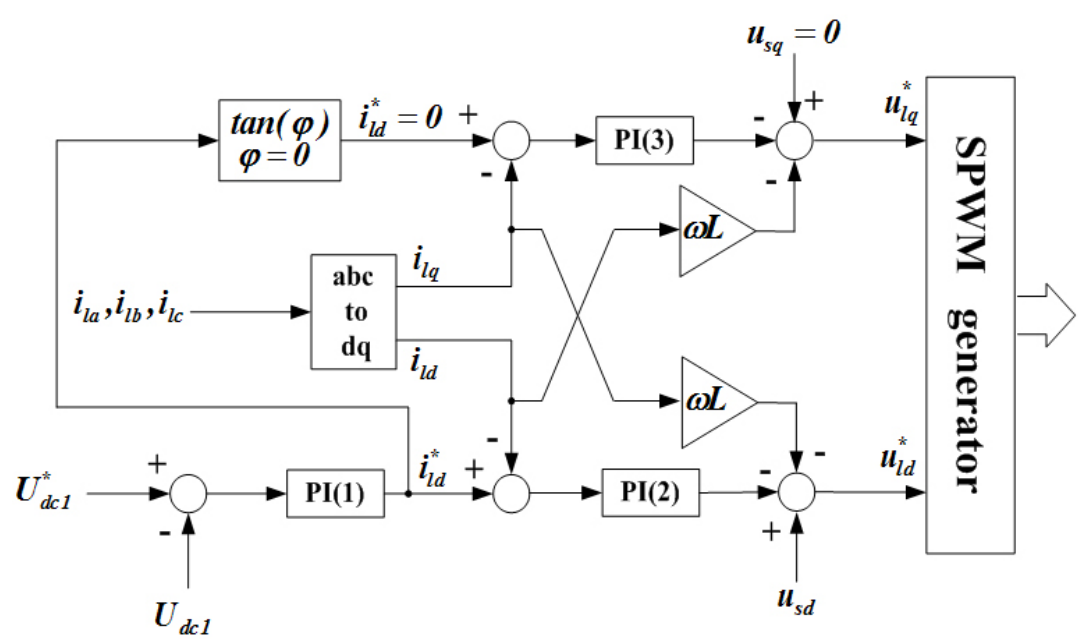

Fig. 5. Control diagram of input stage based on PI controller.

The loads in distribution systems are mostly considered to be passive. Therefore, the constant ac voltage is controlled as an instantaneous feedback amount [16]. The control design of the output stage, based on PI controllers, is shown in Fig. 6.

There are two control loops in this figure. The two signals, including the output effective voltage $\left(U_{o}\right)$ and the instantaneous output voltage $\left(u_{o}\right)$, are considered as feedback signals. The output effective voltage is used for regulating the output loop and the instantaneous output voltage in the inner loop is used for obtaining a sinusoidal output voltage.

Theoretically, the output effective voltage remains constant until the load changes. Moreover, the output voltage can produce a sinusoidal waveform. To avoid phase angle errors, a proportional control (P) is used for the inner loop. It is necessary to note that the three-phase control design is that of a single-phase control. The only different is the $120^{\circ}$ phase angles for the sinusoidal signals.

\section{B. $L Q R$ for a DEPT}

One method for controlling multi-input multi-output (MIMO) systems is using state feedbacks. Nowadays, the design method of a linear quadratic regulator is widely applied as a modern optimal control method [4], [7], [17]. The main task of this method is controlling the static variables in small signal performance. This type of controller is appropriate for multi-input multi-output systems [17], [18]. Since a DEPT is a multi-input multi-output system, an LQR can be used to take control of a 3-wire DEPT to improve its dynamic performance.

The LQR performance index to be minimized is as follows:

$$
J=\frac{1}{2} \int_{0}^{\infty}\left(x_{a}^{T} Q x_{a}+u^{T} R u\right) d t
$$

where the diagonal matrices $Q, R$ define the output and input weights, respectively.

The input control $u$, which is used to minimize the performance index is defined as a feedback, as:

$$
u=-K x_{a}
$$

The gain matrix of the state feedback is defined as:

$$
K=R^{-1} B^{T} P
$$

where $P$ is determined by the Riccati equation

$$
Q-P B R^{-1} B^{T} P+A^{T} P+P A=0 .
$$

The state space model of equations (8) to (11) is suitable for large signal operation in a nonlinear form. Therefore, the corresponding reference amounts can be used. This model is obtained by putting the mentioned equations equal to zero and by replacing all of the variables with their steady states.

The operating points of the nominal steady state include:

$$
\begin{aligned}
& i_{l d}=I_{l d}, i_{l q}=I_{l q}, i_{f d}=I_{f d}, i_{f q}=I_{f q}, i_{L d}=I_{L d}, i_{L q}=I_{L q} \\
& u_{L d}=U_{L d}, u_{L q}=U_{L q}, u_{s}=U_{s}, u_{d c}=U_{d c}, m_{1}=M_{1}, \theta_{1}=\theta_{1}^{*} \\
& m_{2}=M_{2}, \theta_{2}=\theta_{2}^{*}
\end{aligned}
$$

The used small signal model, which has been linearized around operating points, is:

$$
\frac{d \Delta x}{d t}=A . \Delta x+B . \Delta u+E . \Delta v
$$

where:

$$
\begin{aligned}
& \Delta x=\left[\begin{array}{lllllll}
\Delta i_{l d} & \Delta i_{l q} & \Delta u_{d c} & \Delta i_{f d} & \Delta i_{f q} & \Delta u_{L d} & \Delta u_{L q}
\end{array}\right]^{T} \\
& \Delta u=\left[\begin{array}{llll}
\Delta m_{1} & \Delta \theta_{1} & \Delta m_{2} & \Delta \theta_{2}
\end{array}\right]^{T} \\
& \Delta v=\left[\begin{array}{lllll}
\Delta u_{s d} & \Delta u_{s q} & \Delta i_{L d} & \Delta i_{L q}
\end{array}\right]^{T}
\end{aligned}
$$

The non-zero elements of the $A, B, E$ matrix are shown in the appendix.

The LQR is naturally a proportional regulator. Thus, to eliminate the steady state errors in response to a step reference or a disturbance facing the system, an integral optimal regulator is required. The main task of this regulator is finding the optimal input that reduces the state variables to zero with a fast response. Equation (20) is transformed to an optimal integral regulator by adding new variables to the state space.

With regards to the four aims of the DEPT, the four variables $\Delta i_{l d}, \Delta u_{d c}, \Delta u_{L d}, \Delta u_{L q}$ should be controlled and their integrals added to the small signal model. Then the following is obtained: 


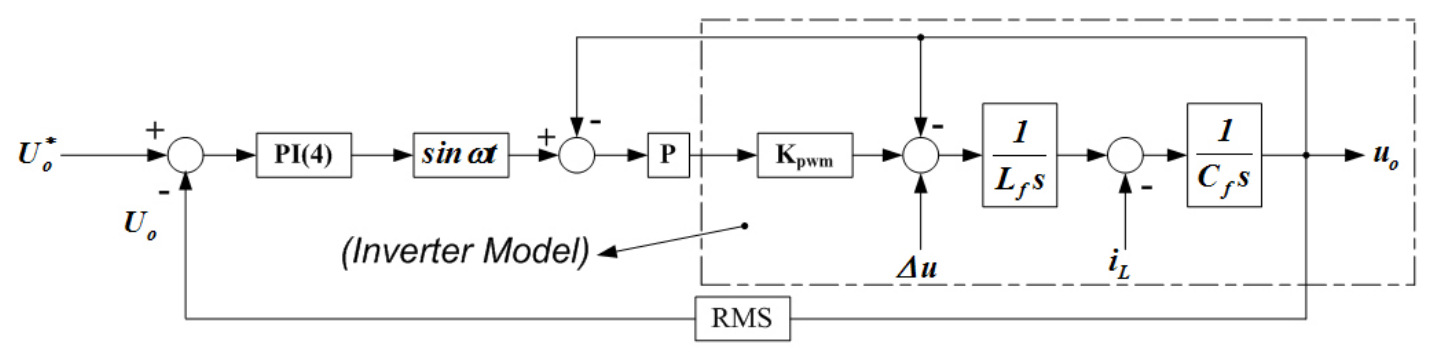

Fig. 6. Control strategy of output stage.

$$
\frac{d \Delta x_{a}}{d t}=A_{a} \cdot \Delta x+B_{a} \cdot \Delta u+E_{a} \cdot \Delta v
$$

where,

$$
\begin{array}{r}
\Delta x_{a}=\left[\Delta i_{l d} \Delta i_{l q} \Delta u_{d c} \Delta i_{f d} \Delta i_{f q} \Delta u_{L d} \Delta u_{L q}\right. \\
\left.\int \Delta i_{l d} \int \Delta u_{d c} \int \Delta u_{L d} \int \Delta u_{L q}\right]^{T}
\end{array}
$$

The non-zero elements of the $A_{a}, B_{a}, E_{a}$ matrices are similar to the $A, B, E$ matrices. Only the following non-zero elements are added to the $A$ matrix:

$$
a_{81}=1, a_{93}=1, a_{106}=1, a_{117}=1
$$

Equation (21) shows that the state variables $\Delta i_{l d}, \Delta u_{d c}$, $\Delta u_{L d}, \Delta u_{L q}$ are close to zero in their steady state. By considering the four control aims of the DEPT, the control system reference amounts can be determined as follows:

$$
I_{l d}=0, U_{L d}=0, U_{L q},=1, U_{d c}=1
$$

Fig. 7 shows the LQR control structure, where $\mathrm{I}$ is the integral operand [19].

\section{SMC for a DEPT}

Sliding mode control is a nonlinear controller that can properly control a system in the presence of structured and unstructured uncertainties. In other words, sliding mode control is one of the most important control methods that possesses non-linear characteristics together with robust properties. This controller has the advantages of non-sensitivity to external disturbances, a fast transient response, design simplicity, simplified execution and reduced calculations. In this method, the control law is usually formed by two separate parts. The first part should provide the state of the system towards the sliding surface. The other part is to keep the system state on the sliding surface.

Consider the following single input dynamic system:

$$
\mathrm{x}^{(n)}=f(X)+b(X) u
$$

where,

$x$ : considered output

$u$ : control input

$f(X), b(X)$ : non-linear functions containing uncertainties with known bounds

$X(t)=\left[\begin{array}{llll}\mathrm{x} & \dot{\mathrm{x}} & \ldots & \mathrm{x}^{(\mathrm{n}-1)}\end{array}\right]^{T}$ : system state vector

If the desired state vector is considered as $X_{d}$, then the following is obtained:

$$
\tilde{X}=X-X_{d}=\left[\begin{array}{llll}
\tilde{\mathrm{X}} & \dot{\tilde{\mathrm{X}}} & \ldots & \tilde{\mathrm{x}}^{(n-1)}
\end{array}\right]^{T}
$$

The control law for $u$ should be in such a way that the state vector $X$ can follow the desired state vector $X_{d}$. The purpose of this activity is so that $\tilde{X}$ will to tend to zero. Therefore, designing the sliding mode control includes the following main parts:

1) Designing a sliding surface for reaching the system requirements: Regarding this matter, a variable surface with time $s(t)$ in the state space $R^{(n)}$ is defined with the scalar equation of $s(X, t)=0$, which is known as the sliding surface or the switching surface [20], [21]:

$$
s(x, t)=\left(\frac{d}{d t}+\lambda\right)^{(n-1)} \tilde{X}
$$

where, $\lambda$ defines the eigenvalue of a closed loop of a system on the sliding surface and is always selected as a positive number.

2) Designing a control law for conducting the system towards the sliding surface: In this method, the control law is established in two parts and each part has special role in controlling the system. The sliding control law all together, is defined as:

$$
u(t)=u_{e q}(t)-u_{n}(t)
$$

The control signal in the sliding mode control should be designed in such a way that the sliding condition exists. For this system, it is assumed that the approximations of the $f(X), b(X)$ function be as $\hat{f}(X), \hat{b}(X)$, so that:

$$
\left\{\begin{array}{l}
\hat{f}=\frac{f_{\max }+f_{\min }}{2} \\
\hat{b}=\left(b_{\min } b_{\max }\right)^{1 / 2}
\end{array}\right.
$$

Hence, the control signal is selected as follows:

$$
u(t)=\frac{1}{\hat{b}}[\hat{u}(t)-k \operatorname{sign}(s)]
$$

Where, $\operatorname{sign}(s)$ is known as the switching function and is as follows:

$$
\operatorname{sign}(s)=\left\{\begin{array}{cc}
1 & s>0 \\
0 & s=0 \\
-1 & s<0
\end{array}\right.
$$

Also, $\hat{u}(t)$ and $k$ are defined as:

$$
\hat{u}(t)=\left[-\hat{f}+\mathrm{x}_{d}^{(n)}-\lambda \tilde{\mathrm{x}}^{(n-1)}-\ldots . \lambda^{(n-1)} \tilde{\mathrm{x}}\right]
$$




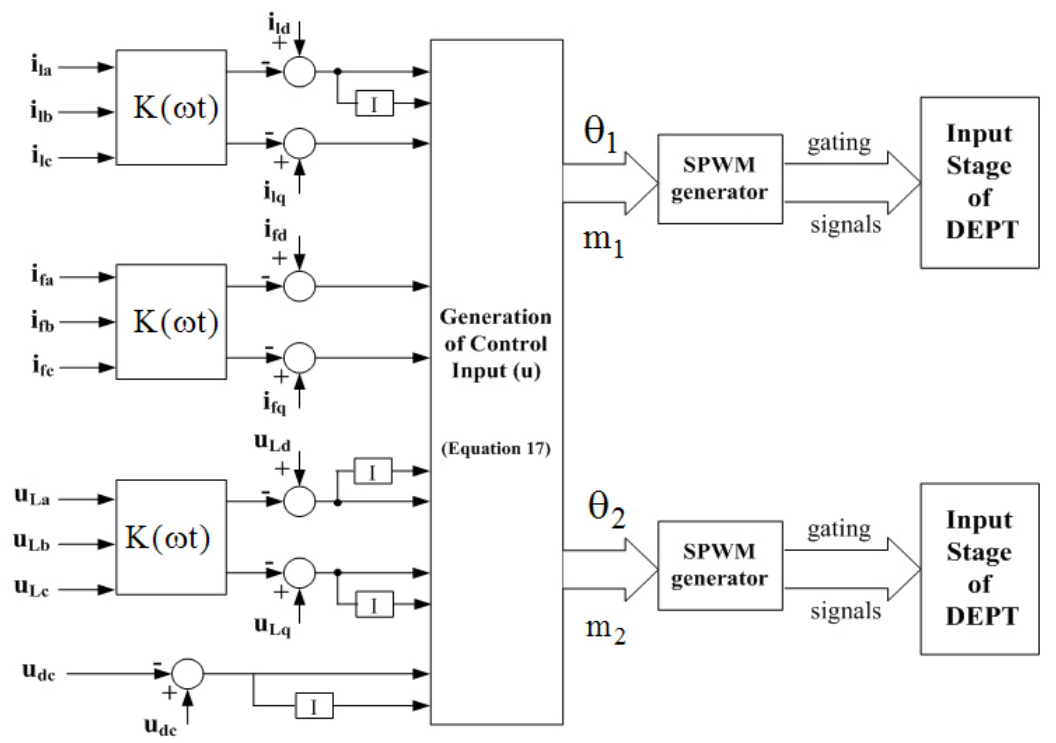

Fig. 7. Control strategy of output stage.

$$
k \geq B(\eta+F)+(B-1)|\hat{u}|
$$

Where $B, F$ are determined as follows:

$$
B=\sqrt{\frac{b_{\max }}{b_{\min }}}, 0<b_{\min }<b<b_{\max } \quad, \quad F \geq|f-\hat{f}|
$$

It is clear that the $\mathrm{k}$ function depends on the system uncertainties amounts and disturbances.

The existence of uncertainties in the system and the limitation of the high switching frequency have effects on the sliding mode control design. This effect is so that the system state should not remain on the switching surface, and vibrate around it which causes a chattering phenomenon to occur. This phenomenon is not appropriate, because it causes an increase in the control activities and excitation of the high frequencies unmodelled dynamics of the system and it may even lead to instability of the system. Thus, a lot of application research has been done to eliminate these problems. Sliding control with fixed boundary layers, variable boundary layers and the integral sliding surface are common methods used to eliminate chattering. The first method, which is used in this paper, is going to be briefly described.

Instead of the $\operatorname{sign}(s)$ function, defined in equation (27), the saturation function should be applied for using the boundary layer in the sliding control. The $\phi$ parameter introduces the thickness of the boundary layer. In other words, the system states in a boundary region are placed around the sliding surface, in this method. The form of this function for a fixed boundary layer, which is equal to $\phi$, is as follows:

$$
\operatorname{sat}\left(\frac{s}{\phi}\right)=\left\{\begin{array}{cc}
1 & s>\phi \\
\frac{s}{\phi} & -\phi \leq s \leq \phi \\
-1 & s<\phi
\end{array}\right.
$$

\section{Proposed SMC Method in Controlling DEPT}

Since a 3-wire DEPT is formed by nonlinear equations, $\mathrm{SMC}$ is used in order to improve its dynamic performance and to obtain suitable steady state behavior.

$i_{l d}, i_{l q}, i_{f d}, i_{f q}$ were selected from equations (8) to (11), as state variables and appropriate state vectors $i_{l d}^{*}, i_{l q}^{*}, i_{f d}^{*}, i_{f q}^{*}$ were considered. To design the SMC, four sliding levels (switching levels) were selected for the system as follow:

$$
\left\{\begin{array}{l}
s_{1}\left(i_{l d}\right)=i_{l d}-i_{l d}^{*} \\
s_{2}\left(i_{l q}\right)=i_{l q}-i_{l q}^{*} \\
s_{3}\left(i_{f d}\right)=i_{f d}-i_{f d}^{*} \\
s_{4}\left(i_{f q}\right)=i_{f q}-i_{f q}^{*}
\end{array}\right.
$$

The control laws are determined by differentiating equation (31) and using the dynamic equation to conduct the system towards the sliding surface. According to the above selected sliding surfaces, four control inputs are obtained as follows:

The first input:

$$
\begin{aligned}
& \dot{s}_{1}\left(i_{l d}\right)=0 \Rightarrow \dot{i}_{l d}-\dot{i}_{l d}^{*}=0 \Rightarrow-(R / L) i_{l d}-\omega i_{l q} \\
& +\left(m_{1} \sin \theta_{1} u_{d c}\right) / L=0 \\
& \Rightarrow m_{1} \sin \theta_{1} u_{d c}=L\left((R / L) i_{l d}+\omega i_{l q}\right)=u_{1}
\end{aligned}
$$

The second input:

$$
\begin{aligned}
& \dot{s}_{2}\left(i_{l q}\right)=0 \Rightarrow \dot{i}_{l q}-\dot{i}_{l q}^{*}=0 \Rightarrow-(R / L) i_{l q}+\omega i_{l d} \\
& -\left(m_{1} \cos \theta_{1} u_{d c}-\sqrt{2} u_{s}\right) / L=0 \\
& \Rightarrow\left(m_{1} \cos \theta_{1} u_{d c}-\sqrt{2} u_{s}\right)=L\left((R / L) i_{l q}-\omega i_{l d}\right)=u_{2}
\end{aligned}
$$

The third input:

$$
\begin{aligned}
& \dot{s}_{3}\left(i_{f d}\right)=0 \Rightarrow \dot{i}_{f d}-\dot{i}_{f d}^{*}=0 \Rightarrow-\omega i_{f d}-\left(u_{L q} / L_{f}\right) \\
& -\left(m_{2} \sin \theta_{2} u_{d c}\right) / L_{f}=0 \\
& \Rightarrow m_{2} \sin \theta_{2} u_{d c}=-L_{f}\left(\omega i_{f d}+\left(u_{L q} / L_{f}\right)\right)=u_{3}
\end{aligned}
$$

The fourth input: 


$$
\begin{aligned}
& \dot{s}_{4}\left(i_{f q}\right)=0 \Rightarrow \dot{i}_{f q}-\dot{i}_{f q}^{*}=0 \Rightarrow \omega i_{f d}-\left(u_{L q} / L_{f}\right) \\
& +\left(m_{2} \cos \theta_{2} u_{d c}\right) / L_{f}=0 \\
& \Rightarrow m_{2} \cos \theta_{2} u_{d c}=L_{f}\left(\left(u_{L q} / L_{f}\right)-\omega i_{f d}\right)=u_{4}
\end{aligned}
$$

Since $i_{l d}^{*}, i_{l q}^{*}, i_{f d}^{*}, i_{f q}^{*}$ are constants, $i_{l d}^{*}, i_{l q}^{*}, i_{f d}^{*}, i_{f q}^{*}$ are equal to zero in equations (32) to (35).

To analyze the DEPT and to evaluate the proposed control system, some disturbances are applied to the system input voltage. The disturbances are in the form of voltage sags, flickers, voltage harmonics and voltage unbalances, hence the existing nonlinear functions are considered for equation (22). To eliminate the disturbances via applying $-k \operatorname{sign}(s)$ to the system, the inputs $u_{1}$ to $u_{4}$ are formed as follows:

$$
\left\{\begin{aligned}
u_{1} & =-k_{1} \operatorname{sign}\left(s_{1}\right) \\
& \Rightarrow m_{1} \sin \theta_{1} u_{d c}=-k_{1} \operatorname{sign}\left(s_{1} / \phi_{1}\right) \\
u_{2} & =-k_{2} \operatorname{sign}\left(s_{2}\right) \\
& \Rightarrow m_{1} \cos \theta_{1} u_{d c}-\sqrt{2} u_{s}=-k_{2} \operatorname{sign}\left(s_{2} / \phi_{2}\right) \\
u_{3} & =-k_{3} \operatorname{sign}\left(s_{3}\right) \\
& \Rightarrow m_{2} \sin \theta_{2} u_{d c}=-k_{3} \operatorname{sign}\left(s_{3} / \phi_{3}\right) \\
u_{4} & =-k_{4} \operatorname{sign}\left(s_{4}\right) \\
& \Rightarrow m_{2} \cos \theta_{2} u_{d c}=-k_{4} \operatorname{sign}\left(s_{4} / \phi_{4}\right)
\end{aligned}\right.
$$

The chattering phenomenon is faced a consideration of equation (36). Thus, to eliminate this phenomenon, the boundary layer in the sliding mode control is used and the function "sat $(s / \phi)$ " replaces "sign $(s)$." Therefore, the system input is re-written by the use of the $s a t(s / \phi)$ function as follows:

$$
\left\{\begin{aligned}
u_{1} & =-k_{1} \operatorname{sat}\left(s_{1} / \phi_{1}\right) \\
& \Rightarrow m_{1} \sin \theta_{1} u_{d c}=-k_{1} \operatorname{sat}\left(s_{1} / \phi_{1}\right) \\
u_{2} & =-k_{2} \operatorname{sat}\left(s_{2} / \phi_{2}\right) \\
& \Rightarrow m_{1} \cos \theta_{1} u_{d c}-\sqrt{2} u_{s}=-k_{2} \operatorname{sat}\left(s_{2} / \phi_{2}\right) \\
u_{3} & =-k_{3} \operatorname{sat}\left(s_{3} / \phi_{3}\right) \\
& \Rightarrow m_{2} \sin \theta_{2} u_{d c}=-k_{3} \operatorname{sat}\left(s_{3} / \phi_{3}\right) \\
u_{4} & =-k_{4} \operatorname{sat}\left(s_{4} / \phi_{4}\right) \\
& \Rightarrow m_{2} \cos \theta_{2} u_{d c}=-k_{4} \operatorname{sat}\left(s_{4} / \phi_{4}\right)
\end{aligned}\right.
$$

By accurate regulation of $k_{1}, k_{2}, k_{3}, k_{4}$ the disturbance to the distribution system can be reduced, and the parameters " $m_{1}, \theta_{1}, m_{2}, \theta_{2}$ " can be obtained, which are required for the SPWM block. Fig. 8 shows the proposed sliding mode control.

In this figure, regarding equation (37), the considered control system inputs are generated. It is worth mentioning that the system inputs include " $m_{1}, \theta_{1}, m_{2}, \theta_{2}$," which should be separated from each other, in a proper way.

The $m_{1}, \theta_{1}$ signals to the first SPWM block and the $m_{2}, \theta_{2}$ signals to the second SPWM block are applied, so the blocks can generate signals related to the DEPT input stage converter gates and the DEPT output stage converter, respectively.

Stability Analysis of the Propose SMC: In order to evaluate the stability of this method, it should be verified that the sliding surface is an absorbent surface such that the system states always move towards it. For this purpose, the Lyapunov's theorem is used by considering the Lyapunov function candidate as follows:

$$
V(s)=\frac{1}{2} s^{2} .
$$

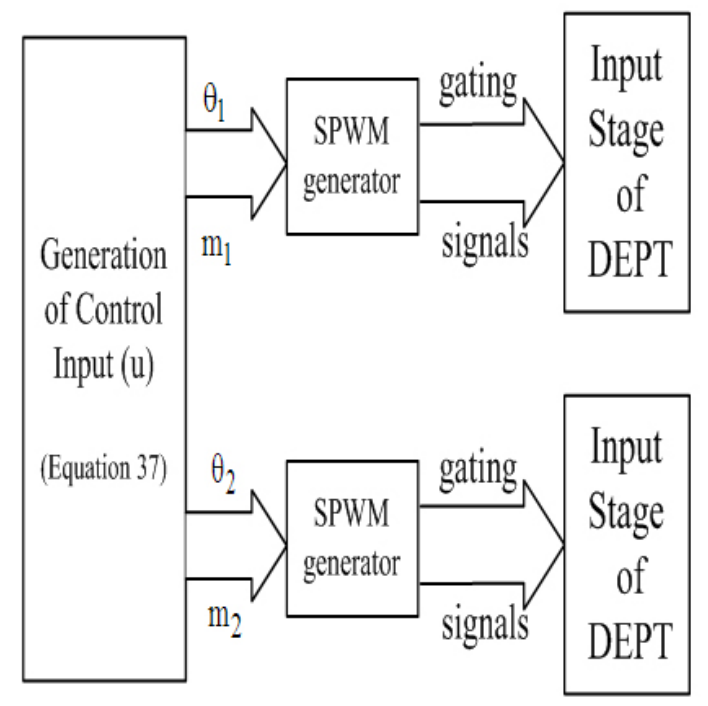

Fig. 8. The proposed sliding mode control.

According to Lyapunov's direct theorem, the stability condition is presented as follows:

$$
\dot{V}(s)=\frac{1}{2} \frac{d}{d t}\left(s^{2}\right)=s \dot{s}<0 .
$$

This equation is called the sliding condition as well. Since $V(s)$ is clearly positive definite, if $\dot{V}(s)$ is negative definite, i.e., the sliding condition is satisfied, then the equilibrium at the origin $s=0$ is globally asymptotically stable. Therefore, $s$ tends to zero as the time tends to infinity. Moreover, all of the trajectories starting off the sliding surface $\mathrm{s}=0$ must reach it in a finite time and then remain on this surface. The control signal must be realized as this sliding condition is provided in the presence of the parameter nonlinearity and uncertainty. By replacing the related equations in the above mentioned condition and using the proposed control law and some mathematical manipulations, the sliding condition is satisfied, if the coefficients $k_{1}, k_{2}, k_{3}, k_{4}$ are selected properly in high values as follows:

$$
\left\{\begin{array}{c}
L\left((R / L) i_{l d}+\omega i_{l q}\right)=-k_{1} \operatorname{sat}\left(s_{1} / \phi_{1}\right) \\
\Rightarrow k_{1}=-L\left((R / L) i_{l d}+\omega i_{l q}\right) / \operatorname{sat}\left(s_{1} / \phi_{1}\right) \\
L\left((R / L) i_{l q}-\omega i_{l d}\right)=-k_{2} \operatorname{sat}\left(s_{2} / \phi_{2}\right) \\
\Rightarrow k_{2}=-L\left((R / L) i_{l q}-\omega i_{l d}\right) / \operatorname{sat}\left(s_{2} / \phi_{2}\right) \\
-L_{f}\left(\omega i_{f d}+\left(u_{L q} / L_{f}\right)=-k_{3} \operatorname{sat}\left(s_{3} / \phi_{3}\right)\right. \\
\Rightarrow k_{3}=L_{f}\left(\omega i_{f d}+\left(u_{L q} / L_{f}\right)\right) / \operatorname{sat}\left(s_{3} / \phi_{3}\right) \\
L_{f}\left(\left(u_{L q} / L_{f}\right)-\omega i_{f d}\right)=-k_{4} \operatorname{sat}\left(s_{4} / \phi_{4}\right) \\
\Rightarrow k_{4}=-L_{f}\left(\left(u_{L q} / L_{f}\right)-\omega i_{f d}\right) / \operatorname{sat}\left(s_{4} / \phi_{4}\right) .
\end{array}\right.
$$

\section{Simulation Results}

In this section, the results of the proposed SMC on DEPT performance are presented. To express the capabilities of the proposed method, the results will be compared with those of a PI controller and a LQR. It is worth mentioning that the forms of the input voltage, the DC-link voltage and the output voltage are obtained by MATLAB/SIMULINK software. The DEPT parameters are indicated in Table I. 
TABLE I

BASIC PARAMETERS OF DEPT

\begin{tabular}{|c|c|}
\hline input voltages & $10 \mathrm{KV}$ \\
\hline output voltages & $400 \mathrm{~V}$ \\
\hline EPT's rated capacity & $500 \mathrm{KVA}$ \\
\hline frequency of network & $50 \mathrm{~Hz}$ \\
\hline frequency of MFT & $1000 \mathrm{~Hz}$ \\
\hline capacitor of input stage & $1000 \mu \mathrm{F}$ \\
\hline input inductor & $10 \mathrm{mH}$ \\
\hline filter capacitor & $800 \mu \mathrm{F}$ \\
\hline filter inductor & $0.24 \mathrm{mH}$ \\
\hline load capacity & $500 \mathrm{KVA} @ 0.8$ \\
\hline
\end{tabular}

\section{A. Generation an unbalance in the input voltage}

It is assumed in this situation that an unbalanced in the voltage is generated in the phases. Fig. 9 shows the simulation results regarding a case where an unbalance voltage occurred in the input, at 0.15 to $0.2 \mathrm{sec}$. In this case, the voltage amplitude of phase A has about a $\% 70$ voltage sag. The frequency of phase B is adjusted to $49 \mathrm{~Hz}$ and the angle of phase $\mathrm{C}$ is $80^{\circ}$. As can be seen in this figure, the input is a three-phase voltage with an intense unbalanced condition. However, the three-phase output voltage is in sinusoidal and symmetrical forms, as a result of using the proposed control process.

\section{B. Flicker generation in the input voltage}

In this case, the input has a sinusoidal wave shape with a frequency of $10 \mathrm{~Hz}$ and a modulation index of $\% 10$. Fig. 10 shows the waveforms under a voltage flicker. This flicker occurs in the input voltage at 0.15 to $0.2 \mathrm{sec}$. As can be seen, the flicker is eliminated in the output voltage and therefore, this voltage has no flicker. Also, the DC-link voltage reaches its real amount (about $20 \mathrm{kV}$ ) after applying the flicker.

\section{Generation of voltage sag in the input}

It is assumed in this case that the three-phase input faces a voltage sag of about \% 40 . Fig. 11 shows the simulation results under such voltage sags at 0.5 to $0.2 \mathrm{sec}$. From this figure, it can be seen that by using the proposed SMC, the output voltage remains fixed and sinusoidal, despite the primary voltage sag. Also, the DC-link voltage reaches the previous constant amount, before the application of a voltage sag at $0.23 \mathrm{sec}$.

\section{Distortion generation in the input waveform}

It is assumed in this situation that voltage harmonics of the $5^{\text {th }}$ and $7^{\text {th }}$ orders with amplitudes of $\% 10$ and $\% 8$, respectively, are applied on the input voltage. Fig. 12 shows the result of the simulation of this situation, with consideration of the proposed SMC. As can be seen in this figure, despite the high amplitudes of the harmonics, the voltage is completely sinusoidal and symmetrical. Also, the DC-link voltage reaches a fixed amount of $20 \mathrm{kV}$, at $0.19 \mathrm{sec}$.

\section{E. Generation of a voltage swell in the input}

It is assumed in this case that the three-phase input faces a voltage swelling of about \%40. Fig. 13 shows the waveforms under voltage swelling at 0.15 to $0.2 \mathrm{sec}$.

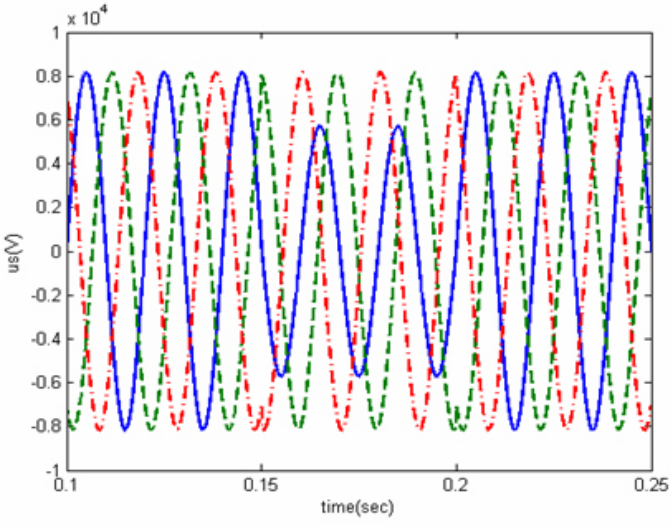

(a)

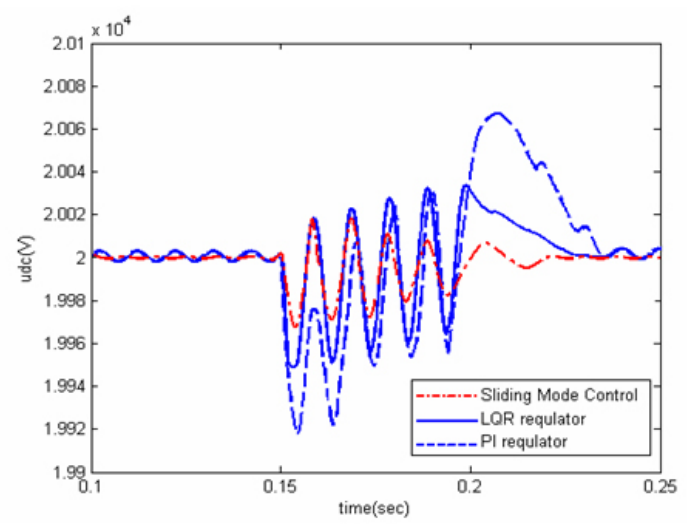

(b)

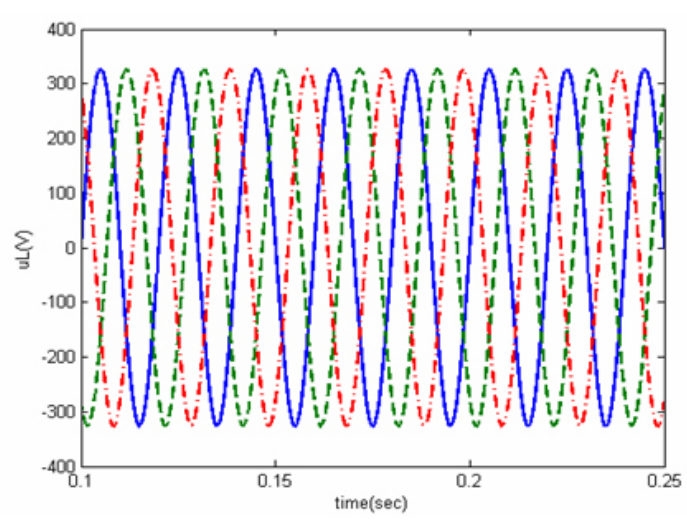

(c)

Fig. 9. Waveforms under unbalance voltage. (a) Input voltages. (b) DC-link voltage. (c) output voltages.

From this figure, it can be seen that by using the proposed SMC, the output voltage remains fixed and sinusoidal. Also, the DC-link voltage reaches $20 \mathrm{kV}$ after applying the swelling. As can be seen, the swelling is eliminated in the output voltage and therefore, this voltage has no swelling.

The DC-link voltage reaching its real value faster indicates that the considered controller responds faster to distribution system disturbances and is on the point of reducing the effects of these disturbances in the DC-link. Hence, from Figs. 10 to 13 (b), it can be seen that:

1- A DC-link with a linear quadratic regulator is preferred over the PI controller.

2- A DC-link with the sliding mode controller is preferred 


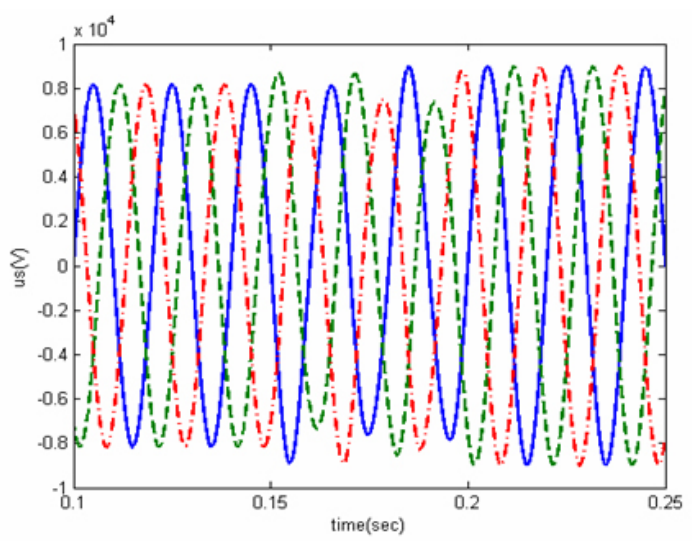

(a)

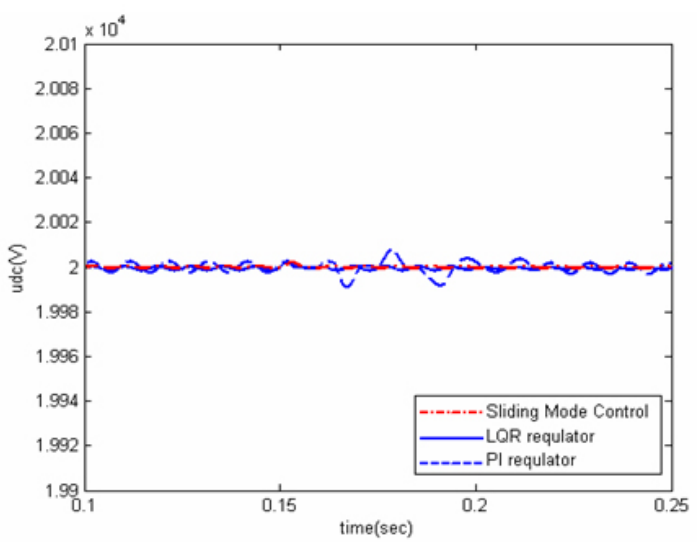

(b)

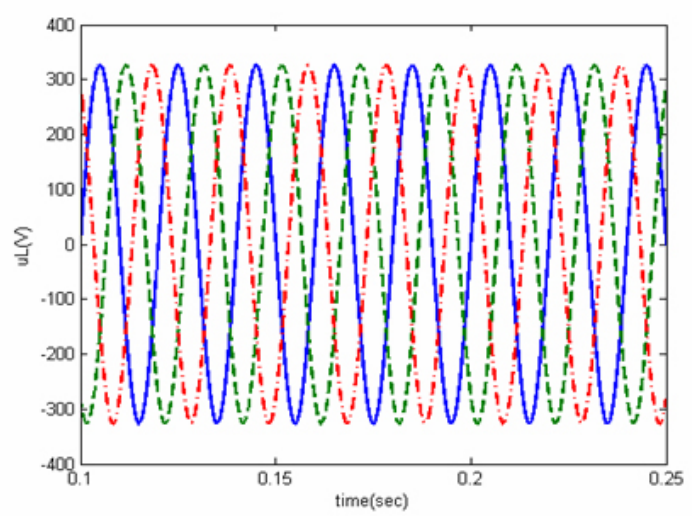

(c)

Fig. 10. Waveforms under voltage flicker. (a) input voltages. (b) DC-link voltage. (c) output voltages.

over the linear quadratic regulator.

The quantitative comparisons of the DC-link voltages, which are presented in Figs. 10(b) to 13(b), are provided in table 2. These results show that the system response overshoot in the case of using proposed SMC in comparison with LQR and PI methods is less. Moreover, by using the proposed SMC, the steady state error reaches to zero. However with other methods, this error does not vanish, which shows the other advantage of the proposed strategy.

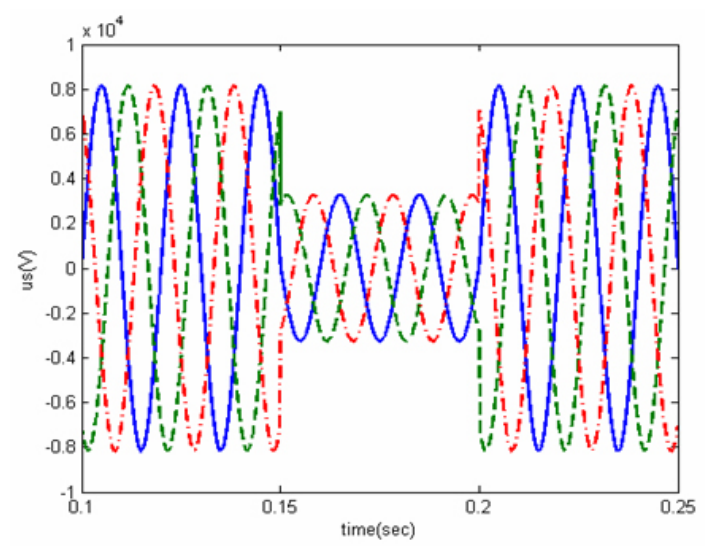

(a)

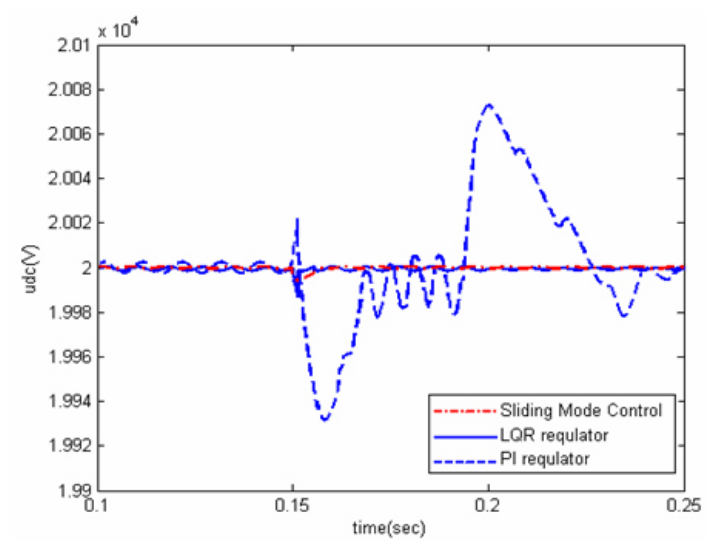

(b)

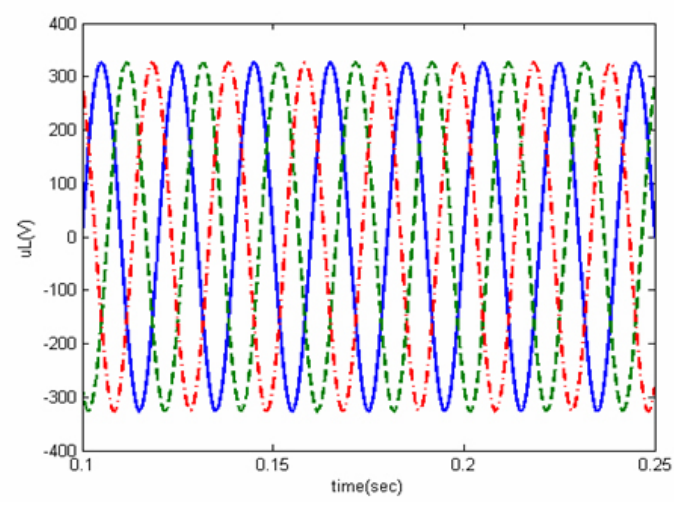

(c)

Fig. 11. Waveforms under voltage sags. (a) input voltages. (b) DC-link voltage. (c) output voltages.

\section{CONCLUSIONS}

In this paper, a 3-wire three-phase DEPT structure was primarily considered. Then to improve the DEPT dynamic performance, a design for a control system was proposed on the basis of the SMC. Also, to compare the proposed controller performance, the SMC method was compared with the control methods of PI and LQR. In the sliding mode control disturbances of the distribution system were rejected by four sliding surface and a proper control law. The simulation results show that the DEPT performance has an appropriate dynamic and steady state performance as a result of using the SMC design, when compared to the PI and LQR controllers. Also, the 


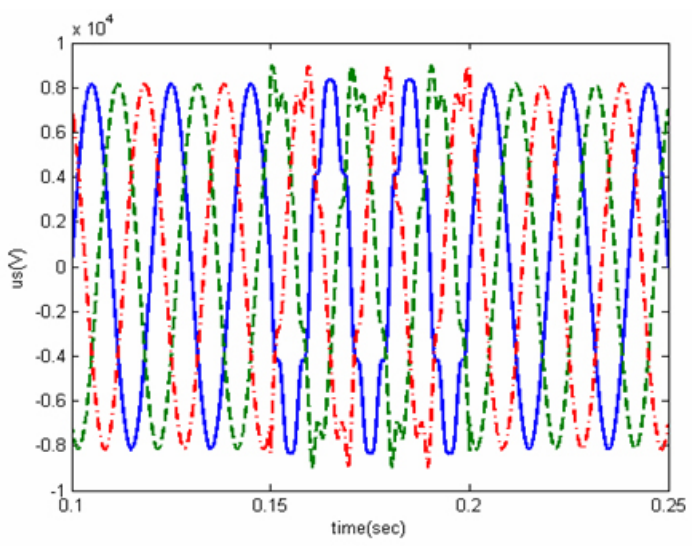

(a)

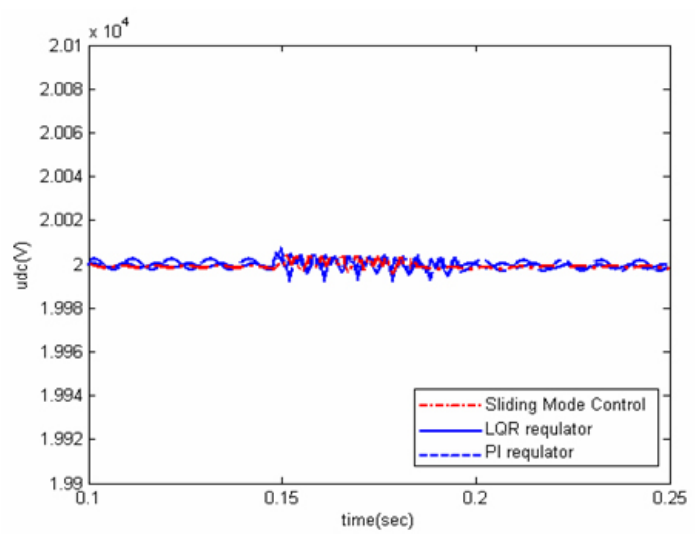

(b)

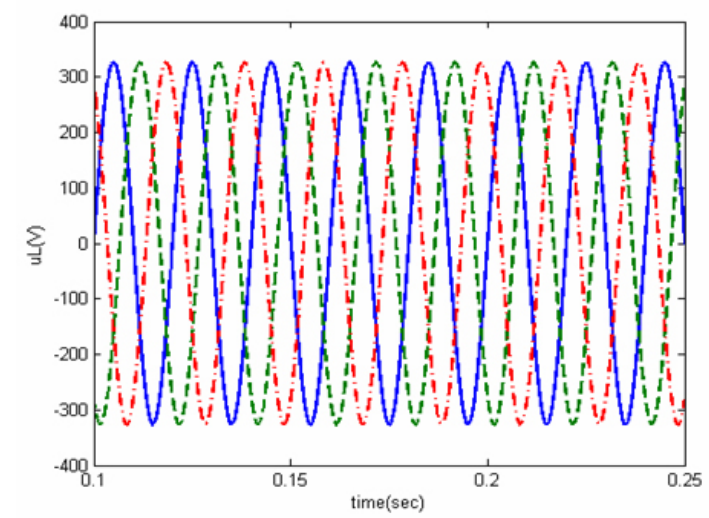

(c)

Fig. 12. Waveforms under voltage harmonics. (a) input voltages. (b) DC-link voltage. (c) output voltages.

SMC for the three-stages of input, isolation and output show a proper response towards each of the quality power factor in the distribution network and it guarantees an improvement in the DEPT dynamic performance. Moreover, the DC-link voltage with the SMC reaches its reference amount (i.e 20kv) faster when compared with the PI controller and the linear quadratic regulator, which shows the priority of the SMC when compared with the other two controllers.

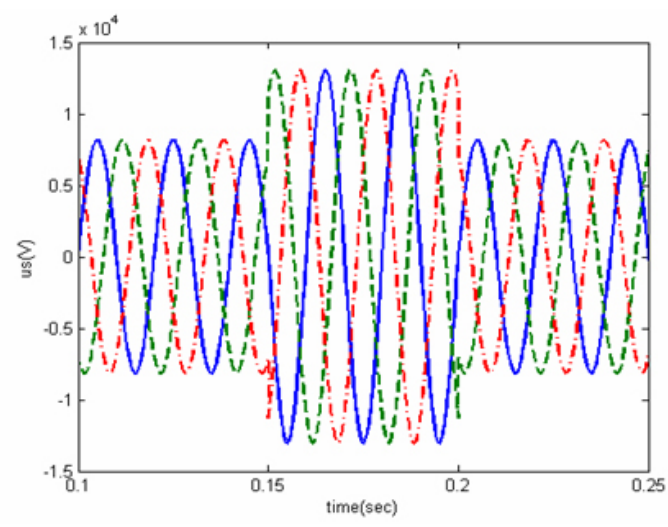

(a)

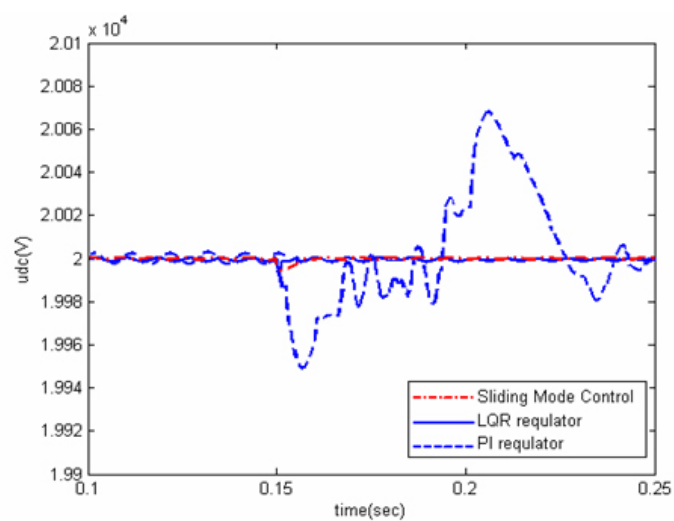

(b)

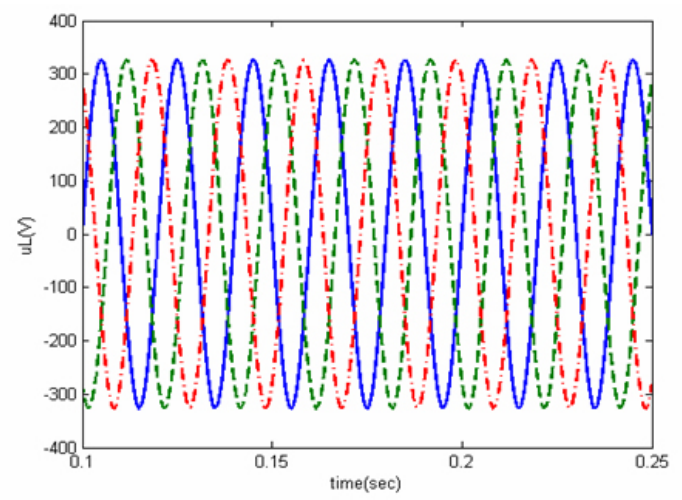

(c)

Fig. 13. Waveforms under voltage swelling. (a) input voltages. (b) DC-link voltage. )c) output voltages.

\section{APPENDIX}

Non-zero elements of matrix $A$ include:

$$
\begin{aligned}
& a_{11}=-\frac{R}{L}, a_{12}=-\omega, a_{13}=\frac{M_{1} \sin \theta_{1}^{*}}{L_{1}}, a_{21}=\omega, a_{22}=-\frac{R}{L} \\
& , a_{23}=-\frac{M_{1} \sin \theta_{1}^{*}}{L}, a_{31}=-\frac{3 M_{1} \sin \theta_{1}^{*}}{2 C}, a_{32}=\frac{3 M_{1} \cos \theta_{1}^{*}}{2 C_{1}} \\
& , a_{34}=\frac{3 M_{2} \sin \theta_{2}^{*}}{2 C}, a_{35}=-\frac{3 M_{2} \cos \theta_{2}^{*}}{2 C}, a_{43}=-\frac{M_{2} \sin \theta_{2}^{*}}{L_{f}} \\
& , a_{45}=-\omega, a_{46}=\frac{1}{L_{f}}, a_{53}=\frac{M_{2} \cos \theta_{2}^{*}}{L_{f}}, a_{54}=\omega, a_{57}=-\frac{1}{L_{f}} \\
& , a_{64}=\frac{1}{C_{f}}, a_{67}=-\omega, a_{75}=\frac{1}{C_{f}}, a_{76}=\omega
\end{aligned}
$$


TABLE II

COMPARISON TABLE BETWEEN DC-LINK VOLTAGES

\begin{tabular}{|c|c|c|c|c|c|}
\hline value & Fig.9.b & Fig.10.b & Fig.11.b & Fig.12.b & Fig.13.b \\
\hline overshoot & $\mathrm{PI}=20.068 \mathrm{kV}$ & $\mathrm{PI}=20.008 \mathrm{kV}$ & $\mathrm{PI}=20.074 \mathrm{kV}$ & $\mathrm{PI}=20.009 \mathrm{kV}$ & $\mathrm{PI}=20.070 \mathrm{kV}$ \\
& $\mathrm{LQR}=20.035 \mathrm{kV}$ & $\mathrm{LQR}=20.004 \mathrm{kV}$ & $\mathrm{LQR}=20.010 \mathrm{kV}$ & $\mathrm{LQR}=20.007 \mathrm{kV}$ & $\mathrm{LQR}=20.006 \mathrm{kV}$ \\
& $\mathrm{SMC}=20.021 \mathrm{kV}$ & $\mathrm{SMC}=20.002 \mathrm{kV}$ & $\mathrm{SMC}=20.003 \mathrm{kV}$ & $\mathrm{SMC}=20.005 \mathrm{kV}$ & $\mathrm{SMC}=20.002 \mathrm{kV}$ \\
\hline steady state & $\mathrm{PI}=20.007 \mathrm{kV}$ & $\mathrm{PI}=20.004 \mathrm{kV}$ & $\mathrm{PI}=20.003 \mathrm{kV}$ & $\mathrm{PI}=20.0035 \mathrm{kV}$ & $\mathrm{PI}=20.004 \mathrm{kV}$ \\
& $\mathrm{LQR}=20.006 \mathrm{kV}$ & $\mathrm{LQR}=20.002 \mathrm{kV}$ & $\mathrm{LQR}=20.002 \mathrm{kV}$ & $\mathrm{LQR}=20.001 \mathrm{kV}$ & $\mathrm{LQR}=20.0018 \mathrm{kV}$ \\
& $\mathrm{SMC}=20 \mathrm{kV}$ & $\mathrm{SMC}=20 \mathrm{kV}$ & $\mathrm{SMC}=20 \mathrm{kV}$ & $\mathrm{SMC}=20 \mathrm{kV}$ & $\mathrm{SMC}=20 \mathrm{kV}$ \\
\hline
\end{tabular}

Non-zero elements of matrix $B$ include:

$b_{11}=\frac{U_{d c} \sin \theta_{1}^{*}}{L}, b_{12}=\frac{U_{d c} M_{1} \cos \theta_{1}^{*}}{L}, b_{21}=-\frac{U_{d c} \cos \theta_{1}^{*}}{L}$
$b_{22}=\frac{U_{d c} M_{1} \sin \theta_{1}^{*}}{L}, b_{31}=\frac{3\left(I_{l q} \cos \theta_{1}^{*}-I_{l d} \sin \theta_{1}^{*}\right)}{2 C}$

$b_{32}=-\frac{3 M_{1}\left(I_{l d} \cos \theta_{1}^{*}+I_{l q} \sin \theta_{1}^{*}\right)}{2 C}$

$b_{33}=\frac{3\left(I_{f d} \sin \theta_{2}^{*}-I_{f q}^{2 C} \cos \theta_{2}^{*}\right)}{2 C}$

$b_{34}=\frac{3 M_{2}\left(I_{f d} \sin \theta_{2}^{*}+I_{f q} \sin \theta_{2}^{*}\right)}{2 C}, b_{43}=-\frac{U_{d c} \sin \theta_{2}^{*}}{L_{f}}$

$b_{44}=-\frac{M_{2} U_{d c} \cos \theta_{2}^{*}}{L_{f}}, b_{53}=\frac{M_{2} \cos \theta_{2}^{*}}{L_{f}}, b_{54}=-\frac{M_{2} U_{d c} \sin \theta_{2}^{*}}{L_{f}}$

Non-zero elements of matrix $E$ include:

$$
e_{11}=\frac{1}{L}, e_{22}=\frac{1}{L}, e_{63}=-\frac{1}{C_{f}}, e_{74}=-\frac{1}{C_{f}}
$$

\section{REFERENCES}

[1] M. D. Manjrekar, R. Kiefemdorf, and G. Venkataramanan, "Power electronic transformer for utility application," in Proc. 2000 IEEE IAS Annual Meeting, pp. 2496-2502, 2000.

[2] C. Mao, J. Lu, S. Fan, and H. Fang, "Power electronic transformer," China Patent ZL $02139030.4,2002$.

[3] J. Cheng, C. Mao, S. Fan, and D. Wang, "Principle of electronic power transformer and its simulation study," Electr. Power Autom. Equip, Vol. 24, No. 12, pp. 23-25, 2004.

[4] S. Fan, C. X. Mao, and L. Chen, "Optimal coordinated PET and generator excitation control for power systems," Int. J. Electr. Power Energy Syst, Vol. 28, No. 3, pp. 158-165, Mar. 2006.

[5] D. Wang, C. X. Mao, and J .M. Lu, "Modeling of electronic power transformer and its application to power system," IET Gener, Transm. Distrib., Vol. 6, No. 1, pp. 887-895, Nov. 2007

[6] D. Wang, C. X. Mao, J. M. Lu, and S. Fan, "Electronic power transformer based power quality control method," High Volt. Eng. Vol. 31 , No. 8 , pp. $63-65,2005$.

[7] D. Wang, C. X. Mao, and J. M. Lu, "Coordinated control of EPT and generator excitation system for multidouble-circuit transmission-lines system," IEEE Trans. Power Del., Vol. 23, No. 1, pp. 371-379, Jan. 2008.

[8] E. R. Ronan, S. D. Sudhoff, S. F. Glover, and D. L. Galloway, "A power electronic-based distribution transformer," IEEE Trans. Power Del., Vol. 17, No. 2, pp. 537-543, Apr. 2002.

[9] M. Kang, P. N. Enjeti, and I. J. Pitel, "Analysis and design of electronic transformers for electric power distribution system," IEEE Trans. on Power Electronics, Vol. 14, No. 6, pp. 1133-1141, Nov. 1999.

[10] D. Wang, C. X. Mao, J. M. Lu, S. Fan, and F. Z. Peng, "Theory and application of distribution electric power transformer," Electr. Power Syst. Res, Vol. 77, No. 3-4, pp. 219-226, 2007.

[11] H. B. Liu, C. X. Mao, J. M. Lu, and D. Wang, "Parallel operation of electronic power transformer and conventional transformer," Third International Conference on Electric Utility Deregulation and Restructuring and Power Technologies, pp. 1802-1808, 2008.
[12] M. Marchesoni, R. Novaro, and S. Savio, "AC locomotive conversion systems without heavy transformer: is it a practicable solution," in Proc. 2002 IEEE Int. Symp. Industrial Electronics, pp. 1172-1177, 2002.

[13] K. Harada, F. Anan, K. Yamasaki, M. Jinno, Y. Kawata, T. Nakashima, K. Murata, and H. Sakamoto, "Intelligent transformer," in Proceedings of the 1996 IEEE PESC Conference, pp. 1337-1341, 1996.

[14] M. E. Fraser, C. D. Manning, and B. M. Wells, "Transformerless fourwire PWM rectifier and its application in ac-dc-ac converters," IEEE Proc. Electr. Power, Vol. 142, No. 6, pp. 410-416, 1995.

[15] J. Kassakian, M. Schlecht, and G. Verghese, "Principles of Power Electronics," Addison-Wesley, 1991.

[16] G. B. Zhang, Z. Xu, and G. Z. Wang, "Steady-state model and its nonlinear control for VSC-HVDC system," in Proceedings of the CSEE, Vol. 22, No. 1, pp. 17-22, 2002

[17] S. Fukuda, Y. Matsumoto, and A. Sagawa, "Optimal-regulator-based control of NPC boost rectifier for unity power factor and reduced neutralpoint-potential variations," IEEE Trans. Ind. Electon., Vol. 46, No. 3, pp. 527-534, Jun. 1999.

[18] D. E. Kirk, "Optimal Control Theory," Prentice-Hall, 2000.

[19] H. B. Liu, C. X. Mao, J. M. Lu, and D. Wang, "Optimal regulatorbased control of electronic power transformer for distribution systems," Electric Power Systems Research, Vol. 79, pp. 863-870, 2009.

[20] L. Jinkun and S. Fuchun, "Research and development on theory and algorithms of sliding mode control," Control Theory and Applications, Chinese, Vol. 24, pp. 407-418, 2007.

[21] D. Zhanfeng and Z. Dongqi, "A hybrid power filter based on sliding mode control," Transactions of China Electrotechnical Society, Chinese, Vol. 17, pp. 92-95, 2002.

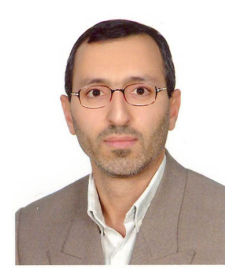

Rahmat-Allah Hooshmand received his B.S. from the University of Mashhad, Iran, in 1989, his M.S. from the University of Tehran, Iran, in 1990, and his Ph.D. from Tarbiat Modarres University, Iran, in 1995, all in Electrical Engineering. He is currently an Associate Professor in the Department of Electrical Engineering, University of Isfahan, Iran. His current research interests include the modeling of power systems and distribution networks.

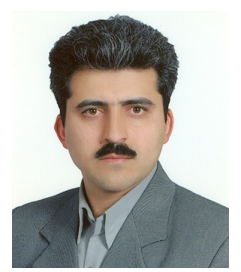

Mohammad Ataei received his B.S. from the Isfahan University of Technology, Iran, in 1994, his M.S. from the Iran University of Science and Technology, Iran, in 1997, and his Ph.D. from the K. N. Toosi University of Technology, Iran, in 2004, (as part of a joint project with the University of Bremen, Germany) all in Control Engineering. Since 2004, he has been with the Department of Electrical Engineering at the University of Isfahan, Iran. His current research interest include control theory and applications in power systems, and chaos control.

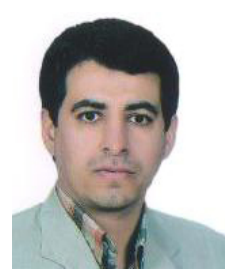

Mohammad Hosein Rezaei was born in Isfahan, Iran, in 1984. He received his B.S. and M.S. in Electrical Engineering from the Islamic Azad University, Najaf Abad Branch, in 2007 and 2010, respectively. His current research interests include electrical machines, nonlinear systems control and sensor less system control. 